Western University

Scholarship@Western

Aboriginal Policy Research Consortium International (APRCi)

$1-2012$

How and when health-care practitioners in Aboriginal Community Controlled Health Services deliver alcohol screening and brief intervention, and why they don't: A qualitative study

Anton Clifford

Anthony Shakeshaft

Catherine Deans

Follow this and additional works at: https://ir.lib.uwo.ca/aprci

Part of the Public Health Education and Promotion Commons, and the Substance Abuse and Addiction Commons

Citation of this paper:

Clifford, Anton; Shakeshaft, Anthony; and Deans, Catherine, "How and when health-care practitioners in Aboriginal Community Controlled Health Services deliver alcohol screening and brief intervention, and why they don't: A qualitative study" (2012).

Aboriginal Policy Research Consortium International (APRCi). 383.

https://ir.lib.uwo.ca/aprci/383 


\title{
How and when health-care practitioners in Aboriginal Community Controlled Health Services deliver alcohol screening and brief intervention, and why they don't: A qualitative study
}

\author{
ANTON CLIFFORD, ANTHONY SHAKESHAFT \& CATHERINE DEANS \\ National Drug and Alcohol Research Centre, Faculty of Medicine, University of New South Wales, Sydney, Australia
}

\begin{abstract}
Introduction. Indigenous Australians experience a disproportionately high burden of alcohol-related harm. Alcohol screening and brief intervention (SBI) offers the potential to reduce this harm if barriers to its delivery in Aboriginal Community Controlled Health Services (ACCHSs) can be optimally targeted. Aims. Examine health-care practitioners' perceptions of, and practices in, alcohol SBI in ACCHSs. Methods. Semi-structured group interviews with 37 purposively selected health staff across five ACCHSs. Results. Alcohol screening independent of standard health assessments was generally selective. The provision of brief intervention was dependent upon factors related to the patient. Four key factors underlying health-care practitioners' perceptions of alcohol SBI were prominent: outcome expectancy; role congruence; utilisation of clinical systems and processes; and options for alcohol referral. Discussion. The influence of outcome expectancy and role congruence on health-care practitioners' alcohol SBI practices has been identified previously, as has to a lesser extent, their less than optimal use of clinical systems and processes. The influence of options for alcohol referral on health-care practitioners' willingness to deliver alcohol SBI primarily related to their misunderstanding of alcohol SBI and the lack of culturally appropriate alcohol referral options for their patients. Conclusion. An intervention combining interactive, supportive and reinforcing evidencebased dissemination strategies is most likely required to enhance health-care practitioners' knowledge and skills in alcohol SBI delivery, positively orientate them to their role in its delivery, and facilitate integration of evidence-based alcohol SBI into routine clinical processes and locally available systems. [Clifford A, Shakeshaft A, Deans C. How and when health-care practitioners in Aboriginal Community Controlled Health Services deliver alcohol screening and brief intervention, and why they don't: A qualitative study. Drug Alcohol Rev 2012;31:13-19]
\end{abstract}

Key words: Indigenous, alcohol, brief intervention.

\section{Introduction}

Alcohol misuse has been identified as a major public health issue, which contributes significantly to premature mortality and excess morbidity in the Indigenous Australian population [1-3].

Aboriginal Community Controlled Health Services (ACCHSs) are primary health-care services planned and managed by local Indigenous Australian communities or organisations [4], and have great potential to reduce alcohol-related harm experienced by the Indigenous Australian population [5,6]. ACCHSs typically adopt a team-based approach to health care, employing a diverse range of health-care practitioners, such as general practitioners (GPs), nurses, Aboriginal health workers (AHWs) and allied health workers [4].

Screening and brief intervention (SBI) is a costeffective treatment for reducing alcohol consumption among non-dependent drinkers in primary care [7]. Despite the cost-effectiveness of alcohol SBI, multiple factors have been shown to influence its routine uptake by health-care practitioners in primary care $[8,9]$. These factors can be broadly categorised into those associated with individual health-care practitioners

Anton Clifford PhD, Post-doctoral Research Fellow, Anthony Shakeshaft PhD, Associate Professor, Catherine Deans BPsych, Research Officer. Correspondence to Dr Anton Clifford, National Drug and Alcohol Research Centre, Faculty of Medicine, University of New South Wales, Sydney, NSW 2052, Australia. Tel: +61 029385 0386; Fax: +61 029385 0222; E-mail: a.clifford@unsw.edu.au 
Table 1. Number of health staff recruited across ACCHSs by role and health-care team

Health-care team

\begin{tabular}{|c|c|c|c|c|}
\hline Role & Primary health care & Drug and alcohol & $\begin{array}{l}\text { Social and emotional } \\
\text { well-being }\end{array}$ & $\begin{array}{l}\text { Number of } \\
\text { staff by role }\end{array}$ \\
\hline AHW & 5 & - & 8 & 13 \\
\hline $\mathrm{RN}$ & 5 & - & - & 5 \\
\hline Team supervisor & 3 & 1 & 1 & 5 \\
\hline GP & 4 & 一 & - & 4 \\
\hline D\&A worker & - & 3 & - & 3 \\
\hline Service manager & 1 & 1 & 1 & 3 \\
\hline \multirow[t]{2}{*}{ Psychologist } & - & 1 & - & 1 \\
\hline & & & & 37 (total) \\
\hline
\end{tabular}

aProvides counselling, social support and advocacy for clients to improve their social, emotional and psychological well-being. ACCHS, Aboriginal Community Controlled Health Service; Admin, administration; AHW, Aboriginal health worker; GP, general practitioner; $\mathrm{RN}$, registered nurse.

(e.g. their perceived lack of time and expertise and preferred approaches for delivering health care), the organisation (e.g. the availability and utilisation of systems and processes to facilitate systematic alcohol treatment and prevention) and the patient (e.g. their preferences for clinical care) [10].

There is general consensus that factors influencing alcohol SBI in specific health-care settings should be clearly identified to ensure that barriers and enablers are optimally targeted $[11,12]$. Indeed, two previous studies implementing alcohol SBI in ACCHSs highlighted the importance of first identifying how factors related to various health-care practitioners, characteristics of the practice setting and the needs and preferences of Indigenous patients, interact within a specific clinical setting to facilitate or inhibit the uptake of alcohol SBI $[13,14]$.

The aim of this present study was to examine the perceptions and practices of health-care practitioners in ACCHSs with regard to alcohol SBI. The findings of this study informed the development of an intervention to improve uptake of alcohol SBI by health-care practitioners in ACCHS settings.

\section{Ethics}

Ethical approval for this study was provided by the Human Research Ethics Committee, University of New South Wales, the Aboriginal Health \& Medical Research Council, Ethics Committee of New South Wales and community Health boards of participating ACCHSs. All study participants provided informed consent.

\section{Setting and participants}

The study was conducted in four rural and one metropolitan ACCHS in the state of New South Wales, Aus- tralia. All ACCHSs were part of a study to disseminate evidence-based alcohol SBI in Indigenous-specific primary health care. Thirty-seven health staff were purposively recruited into the study, $60 \%(n=22)$ of whom worked in a rural ACCHS. The health-care teams and work roles of health staff recruited are summarised in Table 1.

At the time of this study, four ACCHSs had primary care teams in which a GP was not the main provider of health care; only the metropolitan ACCHS had a drug and alcohol team; and rural ACCHS 4 did not have a primary care or drug and alcohol team. The number of various health staff recruited across ACCHSs included: AHW $(n=13)$, registered nurse $(\mathrm{RN})(n=5)$, team supervisor $(n=5)$, GP $(n=4), \mathrm{D} \& \mathrm{~A}$ worker $(n=3)$, Admin $(n=3)$, service manager $(n=3)$ and psychologist $(n=1)$. Sixty-five per cent $(24 / 37)$ of health staff recruited were Indigenous Australian.

\section{Measures}

Health staff members' perceptions of and practices in alcohol SBI.

\section{Methods}

Semi-structured group interviews with health staff of ACCHSs were the primary method used to collect data. Interview questions were informed by the findings of qualitative studies exploring the perceptions of health-care practitioners to alcohol SBI delivery in Indigenous $[13,14]$ and non-Indigenous $[9,15,16]$ primary health-care settings, and notes recorded during field visits to participating ACCHSs. Group interview participants were initially asked how they ask patients 
about alcohol, including what questions they ask and when they ask them, as well as what things make it difficult to ask patients about alcohol. Participants were then asked how they decide if a patient drinks too much alcohol, what they do if a patient drinks too much and what makes it difficult to treat patients who drink too much alcohol. Interviewer prompts were used to follow up questions, focus the discussion and seek clarification of issues.

\section{Procedure}

Step 1. Prior to group interviews, two field visits to each ACCHS were undertaken by one researcher (author 1) to elicit information on the number and composition of health staff and existing primary care systems (e.g. IT systems) and processes (e.g. health assessments). Notes from field visits were organised into themes and informed the interview schedule.

Step 2. Six group interviews with health-care practitioners in primary care and drug and alcohol teams were conducted: one group interview in each of the four rural ACCHSs and two group interviews in the metropolitan ACCHS. Administration and management staff in rural ACCHS 1, 2 and 4 attended group interviews at the request of management in these services. Group size ranged from 3 to 10 participants, with a mean of 7 and median of 6 . Group characteristics varied because of differences between ACCHSs in staff numbers, composition and availability. Group interviews were between 50 and 65 min duration. All group interviews were audiotaped and transcribed verbatim. Transcripts were made available to participants for validation and comment.

\section{Data analysis}

Qualitative data were analysed using The Framework Analysis, a method of qualitative data analysis that begins deductively from pre-defined objectives and is explicit and informed by a priori reasoning [17]. Key steps in the analyses of qualitative data included:

(i) Provisional classification of the content of each transcript using a priori framework (interview questions and study objectives).

(ii) Revision of the framework in response to participants' self-reported perceptions and practices.

(iii) Application of the revised framework to interview transcripts to identify common and salient themes.

(iv) Mapping of themes to identify patterns and associations and possible explanatory factors.

\section{Results}

Current practices in alcohol SBI

Screening. Health-care practitioners typically conducted alcohol screening as a component of standard health assessments, such as the Aboriginal and Torres Strait Islander Health Check, a federally funded health assessment targeting Indigenous Australians. Health-care practitioners from ACCHS 3 reported conducting health assessments with new patients, including alcohol screening using Medical Director (MD), a computerised patient information system developed for clinical settings. Although ACCHSs used a standard set of questions when screening for alcohol as part of health assessments (e.g. Adult Health Checks and Patient Intake Assessments), question domains varied considerably and included, for example, quantifying alcohol consumption to assess risk: How many standard drinks per day? Number of days per week? Type of alcohol consumed? (metro ACCHS); patient self assessment of drinking status: What type of Drinker are you: Periodic Drinker; Reformed Drinker; Chronic Heavy Drinker, Non Drinker, Safe Drinker? (metro ACCHS, drug and alcohol team) and drinking status only: Do you drink alcohol? (rural ACCHS 3).

Alcohol screening independently of health assessments was generally selective; prompted by alcoholrelated presentations, such as injuries and stress-related conditions, as well as disease and illness likely to be caused or worsened by heavy drinking, such as abnormal liver function and diabetes. In these contexts, health-care practitioners typically reported using informal inquiry to assess patients' risk levels, and alcohol consumption was generally not specified or described obscurely in paper and electronic records. No healthcare practitioner reported routinely using the alcohol screening questions in MD. One GP (rural ACCHS 1) with an interest in addiction medicine reported using the CAGE instrument.

Brief intervention. Health-care practitioners were more likely to intervene with patients with risky drinking when: they perceived it would not hinder patient rapport; the presenting patient condition was likely to be alcohol-related; and they perceived the patient to be high risk. GPs and RNs typically reported providing risky drinkers with verbal advice on low-risk drinking guidelines and potential risks and harms, while AHWs were more likely to advise their patients of the potential negative social implications of drinking too much alcohol.

Health-care practitioners' accounts of delivering alcohol brief intervention ranged from using recognised 
BI strategies, such as motivational interviewing, to ineffective strategies, such as admonishing patients who drank too much.

\section{Key factors influencing alcohol SBI}

Four factors influencing health-care practitioners' practices in alcohol SBI were prominent: outcome expectancy; role congruence; utilisation of clinical systems and processes; and perceptions of alcohol referral options.

Outcome expectancy. Health-care practitioners generally had expectations that routine alcohol SBI would lead to a negative outcome for themselves or their patient.

First, routine alcohol screening could lead to more problems than it could solve. GPs in particular expressed concern that asking a patient about their alcohol use would identify multiple and complex problems they had neither the time nor expertise to treat.

One of the reasons I don't really ask whether there is um, alcohol-related problems, like mental health problems and things, is so what...the patient's been drinking in a harmful way, so what? I mean, what can I do for him in my surgery? (GP rural ACCHS 2)

Second, nurses and AHWs expressed concerns that alcohol screening could offend patients and damage rapport.

If someone comes in for a cough and we automatically start asking them about drugs and alcohol then they're going turn around and go back out the door. You've sort of got to build up that rapport with them first before you know what you can and can't get out of them. (RN metro ACCHS)

While damaging patient rapport was a common concern among AHWs and RNs, when asked how patients normally respond to questions about their drinking, no health-care practitioner reported their patients responding in a way that suggested they objected to being asked.

Third, all types of health-care practitioners expressed scepticism as to the effectiveness of alcohol BI: at-risk drinkers were described as attentive but non-responsive to advice to reduce alcohol consumption. General perceptions were that risky drinkers willing to change would change, while those resistant to change would not. This appeared to thwart health-care practitioners' consideration of more subtle issues related to modifying risky drinking behaviour.
To modify their behaviour, I would say (our success) it's minimal. I mean I always say to people it's impacting on your health, cut down. But then what people do when they walk out of here you don't . . . (control) (GP rural ACCHS 3)

Role congruence. No health-care practitioner rejected outright that they had a role in alcohol SBI. However, health-care practitioners' perceptions of how well alcohol SBI fitted within their role appeared to influence their willingness to deliver it. For example, Indigenous health-care practitioners with a defined role in drug and alcohol (D\&A) prevention (e.g. AOD worker) or engaged in a structured process for its delivery (e.g. delivering health assessments) reported greater involvement in alcohol SBI than those with less defined and structured D\&A roles.

I do with health checks, that's (alcohol screening) one of the mandatory components, so yeah, every time anyone has a health check I ask that (alcohol) question ... (AHW rural ACCHS 2)

General perceptions among RNs were that they had a key role in alcohol SBI as part of health assessment processes, but that it was the GP's role to deliver it opportunistically. GPs, however, said they were usually too busy treating the patient's presenting health condition to ask them about their drinking.

Utilisation of clinical systems and processes. Health-care practitioners' utilisation of clinical systems and processes to deliver alcohol SBI appeared less than optimal. Alcohol screening questions were incorporated in forms for the Adult Health Check, Patient Intake Assessments and Patient Care Plans, ensuring their uniformity across health-care practitioners within individual ACCHSs. However, these questions were not validated, nor were they consistent across different forms.

Alcohol SBI delivered opportunistically in standard consultations was not routinely or uniformly documented; AHWs were generally unaware of the alcohol screening function in $\mathrm{MD}$, while GPs and RNs were unaccustomed to using it for this purpose or reported a preference for written documentation in patient progress notes. Alcohol information in electronic and paper records was generally poorly linked and inconsistent, primarily because of different methods of recording by health-care practitioners. Indigenous-specific alcohol SBI guidelines and resources, although available in all ACCHSs, were referred to infrequently.

Alcohol referral options. A lack of appropriate alcohol referral options was identified as a prominent barrier to 
alcohol SBI in all group interviews. Specifically, healthcare practitioners reported a lack of: adequate follow-up support for patients post alcohol rehabilitation; appropriate alcohol detoxification services; AOD and counselling staff; and funding to transport patients to remote rehabilitation and detoxification units. Without accessible and appropriate alcohol detoxification and rehabilitation services for patient treatment and referral, health-care practitioners perceived alcohol SBI to be of little benefit to their patients.

... it's wonderful to have all the latest and greatest resources, up to date information, but unless you have referral pathways that you can refer your patients onto, all the paperwork in the world's not going to do you any good. (RN rural ACCHS 2)

\section{Discussion}

This study has several key limitations. First, qualitative interviews were conducted among small groups of health staff from five ACCHSs. This limits the range of responses obtained and their generalisability to healthcare practitioners in other ACCHSs. Health-care practitioners were, however, from diverse professional backgrounds, had different levels of experience, and worked in ACCHSs that varied in size and function, increasing the potential for interviews to elicit insights from multiple perspectives. Second, interview findings are based on self-report, which may not reflect actual practice and could lead to over-reporting of activities perceived as socially desirable. Third, group interviews raise the potential for the views of more vocal participants to dominate discussion [18]. The potential for discussions to favour or neglect the views of individual participants was moderated by the skills of the interviewer, and respondent validation, which gave all participants the opportunity to review interview transcripts and make additional comments. Finally, the extent to which health-care practitioners' perceptions of factors influencing alcohol SBI are hypothetical or based on experience is unclear.

Despite these limitations, health-care practitioners' preferences for selective alcohol screening and their low provision of brief intervention are consistent with findings of similar studies conducted in mainstream healthcare settings $[8,9,15,16]$. Likewise, a lack of role congruence and low outcome expectancy have been identified as key barriers to the uptake of alcohol SBI [8]. They are also consistent with a number of theoretical domains identified as important for modifying health-care practitioner behaviour, including beliefs about consequences (outcome expectancy) and professional role (role congruence) [19]. For example, it has been estimated that 10 out of every 100 patients screened for alcohol will be at risk drinkers, and that brief intervention is effective for reducing alcohol consumption in one out of every 10 at-risk drinkers [20]. It is therefore likely that some health-care practitioners who screen their patients for alcohol may have limited success in the provision of brief intervention to those at risk. Those that do may be more likely to perceive alcohol SBI as ineffective and therefore less likely to integrate it into their role [10]. Furthermore, the efficacy of alcohol SBI for Indigenous Australians is uncertain [21], possibly contributing to low expectations among health-care practitioners in ACCHSs regarding its acceptability and effectiveness.

Despite the relative lack of evidence to support the choice of strategies to specifically address outcome expectancy and role congruence [22], theoretical models for the implementation of interventions to improve health-care delivery provide some insight into which strategies are likely to be effective [23]. Interactive, supportive and reinforcing strategies tailored to the needs and preferences of health-care practitioners in their specific health-care settings would appear to offer considerable promise [24,25].

Health-care practitioners' low utilisation of clinical systems and process for alcohol SBI is not unique to ACCHSs. Differences between ACCHSs and mainstream primary care, however, raise important considerations for addressing this issue. ACCHSs team-based approach means the doctor is not typically the primary or sole primary care provider, as is the case in mainstream general practice. Computerised patient information systems are cost-effective for prompting health-care delivery across various health-care practitioners $[26,27]$ and in some cases have been shown to improve the quality of patient care [28]. One possible reason for health-care practitioners' less than optimal utilisation of MD for alcohol SBI could relate to it being primarily designed to prompt and capture clinical activities in general practice, not primary health care [29]. This is possibly reflected in that ACCHSs with MD generally use additional electronic systems to prompt and capture their processes of health care, while those with a computerised patient information system designed for primary health care generally do not [30]. Another likely reason is that health-care practitioners in ACCHSs, with perhaps the exception of GPs, probably lack specific training in how to optimally use MD for preventive health care. Strategies to improve health-care practitioners' knowledge and skills in how to use MD for alcohol prevention should therefore ideally include practical training in how to use MD to screen for alcohol, identify at-risk patients for brief intervention and flag high-risk patients for referral and follow up.

Notably, health-care practitioners' perception that a lack of alcohol referral options is a significant barrier to 
alcohol SBI has not been a prominent factor in other studies. Its prominence in this study raises several possibilities. First, drinking patterns of Indigenous patients presenting to ACCHSs may reflect those of the broader Indigenous Australian population in which a disproportionately high proportion of Indigenous persons do not consume alcohol, or do so to high-risk levels with multiple concomitant negative effects [2]. This would explain GPs' concerns of alcohol screening opening up 'multiple and complex problems' they do not have the time nor skills to address, which in turn highlights the need to create a more positive orientation towards alcohol screening in ACCHSs. Thus, health-care practitioners need to believe they can help Indigenous patients to cut down or stop drinking too much alcohol and be made aware that generally, Indigenous patients expect to be asked about their drinking [5]. Second, health-care practitioners' emphasis on the lack of alcohol referral options suggests a lack of knowledge of alcohol SBI. Even when a definition of alcohol SBI was provided, health-care practitioners generally did not recognise it as an effective alcohol treatment in itself. Interactive education, when optimally combined with a supportive and reinforcing strategy, such as outreach support, has proven to be effective for improving health-care practitioners' preventive health-care knowledge and practices [31]. Fourth, health-care practitioners' accounts of a lack of alcohol referral options for their Indigenous patients is consistent with a key finding of a recent report on Indigenous-specific drug and alcohol intervention programs, which found significant gaps in the range and appropriateness of drug and alcohol services available to rural and remote Indigenous Australians [32].

\section{Conclusion}

The qualitative findings from this study and evidence from the research literature suggest that interactive, supportive and reinforcing strategies are most likely required to improve alcohol SBI delivery in ACCHS settings. Ideally, these should include two complementary components: first, interactive education to improve health-care practitioners' knowledge and skills in alcohol SBI delivery and positively orientate them to their role in its delivery, and second, tailored outreach support to facilitate integration of evidence-based alcohol SBI into clinical processes (e.g. patient triage and referral) and systems (e.g. electronic and manual screening templates), and to optimise the use of alcohol referral pathways.

As a sequel to this study, an intervention combining interactive education and tailored outreach support will be implemented in participating ACCHSs and its effec- tiveness for improving uptake of alcohol SBI by healthcare practitioners evaluated.

\section{Acknowledgements}

Thank you to staff from participating ACCHSs for their involvement in this study. We also wish to thank individual practitioners in the drug and alcohol field for delivering training workshops to health-care staff of participating ACCHSs. We gratefully thank the Department of Health \& Ageing (DoHA) for funding this research through the National Drug Research Institute (NDRI), Perth, Australia. Dr Anton Clifford undertook this research as a National Health \& Medical Research Council (NHMRC) Post-doctoral Training Research Fellow in Aboriginal and Torres Strait Islander Health.

\section{References}

[1] Chikritzhs TPR, Gray D, Stearne A, Saggesr S, Jones P. Trends in alcohol-attributable deaths among Indigenous Australians, 1998-2004. National Alcohol Indicators. Perth: National Drug Research Institute, Curtin University of Technology, 2007.

[2] Commonwealth Department of Human Services and Health. National Drug Strategy Household Survey: Urban Aboriginal and Torres Strait Islander Peoples Supplement 1994. Canberra: Australian Government Publishing Service, 1996.

[3] Dempsey KE, Condon CR. Mortality in the northern territory 1979-97. Darwin: Territory Health Services, 1999.

[4] Hunter P, Mayers P, Couzos S, et al.; Aboriginal Community Controlled Health Services. General practice in Australia: 2004. Canberra: Commonwealth Department of Health and Ageing, 2005:337-56.

[5] Hunter E, D'Abbs P. In 'modest but practical ways': medical practitioners and substance misuse in Aboriginal Australians. Intern Med J 2003;33:333-5.

[6] Larkins SL, Geia LK, Panaretto KS. Consultations in general practice and at an Aboriginal community controlled health service: do they differ? Rural and Remote Health 2006;5:1-12.

[7] Kaner EFS, Beyer F, Dickinson HO, et al. Effectiveness of brief alcohol interventions in primary care populations. Cochrane Database Syst Rev 2007;(2): Art. No.: CD004148. DOI: 10.1002/14651858.CD004148.pub3.

[8] Roche AM, Hotham ED, Richmond R. The general practitioner's role in AOD issues: overcoming individual, professional and systemic barriers. Drug Alcohol Rev 2002;21:223-30.

[9] Aalto M, Pekuri P, Seppa K. Obstacles to carrying out brief intervention for heavy drinkers in primary health care: a focus group study. Drug Alcohol Rev 2003;22:169-73.

[10] Roche A, Freeman T. Brief interventions: good in theory but weak in practice. Drug Alcohol Rev 2004;23:11-18.

[11] Babor TF, Higgens-Biddle J, Dauser D, Higgens P, Burleson JA. Alcohol screening and brief intervention in primary care settings: implementation models and predictors. J Stud Alcohol 2005;66:361-8.

[12] Babor TF, Dauser D, Higgens-Biddle JC. Alcohol screening and brief intervention: dissemination strategies for medical practices and public health. Addiction 2000;95: 677-86. 
[13] Brady M, Sibthorpe B, Bailie R, Ball S, Sumnerdodd P. The feasibility and acceptability of introducing brief intervention for alcohol misuse in an urban Aboriginal medical service. Drug Alcohol Rev 2002;21:375-80.

[14] Clifford A, Shakeshaft A. Evidence-based alcohol screening and brief intervention in Aboriginal Community Controlled Health Services: experiences of health-care providers. Drug Alcohol Rev 2010;10:1-8.

[15] Lock CA, Kaner E, Lamont S, Bond S. A qualitative study of nurses' attitudes and practices regarding brief alcohol intervention in primary health care. J Adv Nurs 2002; 39:333-42.

[16] Roche A, Guray C, Saunders JB. General practitioners' experiences of patients with drug and alcohol problems. Br J Addict 1991;86:263-75.

[17] Ritchie J, Spencer L. Qualitative data analysis for applied policy research. In: Bryman A, Burgess RG, eds. Analysing qualitative data. London: Routledge, 1994:173-94.

[18] Fontana A, Frey J. Interviewing the art of science. In: Denzin NK, Lincoln YS, eds. Handbook of qualitative research. Thousand Oaks: Sage, 1994:361-76.

[19] Michie S, Johnston M, Abraham C, Lawton R, Parker D, Walker A. Making psychological theory useful for implementing evidence-based practice: a consensus approach. Qual Saf Health Care 2005;14:26-33.

[20] Proude E. Brief Interventions. In: Australian Government Department of Health and Ageing. Guidelines for the Treatment of Alcohol Problems. Canberra: Commonwealth of Australia; 2009:41-5.

[21] Shakeshaft A, Clifford A, Shakeshaft M. Reducing alcoholrelated harm experienced by Indigenous Australians: identifying opportunities for Aboriginal health services. Aust NZ J Public Health 2010;34:S41-5.

[22] Oxman AD, Thomson MA, Davis DA, Haynes RB. No magic bullets: a systematic review of 102 trials of interventions to improve professional practice. CMAJ 1995;153: 1423-31.

[23] Sanson-Fisher R. Diffusion of innovation theory for clinical change. Med J Aust 2004;180(Suppl.):S55-6.
[24] Kaner EFLC, McAvoy BR, Heather N, Gilvarry E. A RCT of three training and support strategies to encourage implementation of screening and brief alcohol intervention by general practitioners. Br J Gen Pract 1999;49:699-703.

[25] Grol R, Grimshaw J. From best evidence to best practice: effective implementation of change in patients' care. Lancet 2003;362:1125-30.

[26] Shanahan M, Shakeshaft A, Mattick RP. Modelling the costs and outcomes of changing rates of screening for alcohol misuse by general practitioners in the Australian context. Appl Health Econ 2006;5:155-66.

[27] Balas EA, Austin SM, Mitchell JA, Ewigman BG, Bopp $\mathrm{KD}$, Brown GD. The clinical value of computerized information services a review of 98 randomized clinical trials. Arch Fam Med 1996;5:271-8.

[28] Delpierre C, Cuzin L, Fillaux J, Alvarez M, Massip P, Lang T. A systematic review of computer-based patient record systems and quality of care: more randomized trials or a broader approach? Int J Qual Health Care 2004;16:40716.

[29] HCN (Health Communication Network) Medical Director. Online. Available at: http://www.hcn.com.au/Products/ Medical+Director (accessed September 2010).

[30] Office for Aboriginal and Torres Strait Islander Health (OATSIH) and National Aboriginal Community Controlled Health Organisation (NACCHO). A national profile of Australian Government funded Aboriginal and Torres Strait Islander Primary Health Care Services: Service Activity Reporting, 2003-04 Key Results. Canberra: Australian Government Department of Health and Ageing, 2006.

[31] Hulscher $M$, Wensing $M$, Grol $R$, van der Weijden $T$, van Weel C. Interventions to improve the delivery of preventive services in primary care. Am J Public Health 1999;89:73746.

[32] Gray D, Stearne A, Wilson M, Doyle M. Indigenousspecific alcohol and other drug interventions: continuities, changes and areas of greatest need. A report for the National Indigenous Drug and Alcohol Committee. Canberra: Australian National Council on Drugs, 2010. 